\title{
The ratio of serum placenta growth factor to soluble vascular endothelial growth factor receptor-1 predicts the prognosis of hepatocellular carcinoma
}

\author{
SAKAE NAGAOKA ${ }^{1}$, TAKAFUMI YOSHIDA ${ }^{1}$, JUNJI AKIYOSHI ${ }^{1}$, JUN AKIBA $^{2}$, TAKAO HISAMOTO ${ }^{1}$, \\ YOKO YOSHIDA ${ }^{1}$, MITSUHIKO ABE ${ }^{1}$, HIRONORI KOGA ${ }^{1}$, TAKUJI TOIRIMURA ${ }^{1}$, \\ TAKATO UENO $^{1}$ and MICHIO SATA ${ }^{1}$ \\ ${ }^{1}$ Division of Gastroenterology, Department of Medicine, Liver Cancer Division, \\ Research Center for Innovative Cancer Therapy and Center of the 21st Century COE Program for Medical Science, \\ ${ }^{2}$ Department of Pathology, Kurume University School of Medicine, 67 Asahi-machi, Kurume 830-0011, Japan
}

Received October 30, 2009; Accepted December 28, 2009

DOI: 10.3892/or_00000807

\begin{abstract}
Angiogenesis plays crucial roles in development and progression of hepatocellular carcinoma (HCC). Placenta growth factor (PLGF), belonging to vascular endothelial growth factor (VEGF) family, is involved in angiogenesis associated with cancer. Soluble VEGF receptor-1 (sVEGFR1) has been thought to be an intrinsic negative regulator for PLGF. We investigated whether serum PLGF and serum sVEGFR1 is associated with prognosis of HCC. Serum PLGF and sVEGFR1 levels were measured in 145 patients with HCC using enzyme-linked immunosorbent assay. The levels of these factors and the ratio of PLGF to sVEGFR1 were analyzed in relation with clinical parameters. The higher level of sVEGFR1 and the lower ratio of PLGF/sVEGFR1 were significantly associated with poor survival in HCC. Cox regression analysis revealed that the lower ratio of PLGF/sVEGFR1 independently correlated to prognosis of patients with HCC. The ratio of PLGF/sVEGFR1 was independent prognostic indicator for HCC. The ratio of PLGF/sVEGFR1 should be addressed in anti-angiogenic therapy for HCC.
\end{abstract}

\section{Introduction}

Hepatocellular carcinoma (HCC) is one of the most common malignancies $(1,2)$. HCC is characterized by hypervascularity mainly supplied by hepatic artery. Angiogenesis is thought to play a pivotal role in the progression of HCC. Hypervascularity which is promoted in proportion to dedifferentiation of HCC is regarded as one of the marker for invasiveness and metastasis (3).

Correspondence to: Dr Takafumi Yoshida, Division of Gastroenterology, Department of Medicine, Kurume University School of Medicine, 67 Asahi-machi, Kurume 830-0011, Japan

E-mail: tayoshi@med.kurume-u.ac.jp

Key words: serum placenta growth factor, vascular endothelial growth factor receptor-1, hepatocellular carcinoma
Tumor angiogenesis is regulated by a variety of proangiogenetic and anti-angiogenetic factors. Vascular endothelial growth factor (VEGF) has been recognized to be an essential proangiogenetic factor in HCC (4). However, it remains controversial that the expression of VEGF in tumor and serum is directly correlated to vascularity, invasiveness, metastasis and prognosis of $\operatorname{HCC}(5,6)$. These findings suggest that other proangiogenic or anti-angiogenic factors may also be involved in angiogenesis of HCC.

Placenta growth factor (PLGF) is a proangiogenetic factor, belonging to the VEGF family. PLGF is not expressed in normal human tissues except the placenta and trophoblasts (7). PLGF and VEGF have their own specific functions in angiogenesis. PLGF is of little importance in the development of a fetus and in normal growth processes (8). Impairment of PLGF, in contrast to VEGF, does not affect healthy vessels but diminish angiogenesis in cancer and ischemia $(9,10)$. PLGF thus plays a crucial role in pathological angiogenesis. It has been reported that PLGF level in tumors correlate with tumor stage, vascularity, recurrence and poor outcome in many cancers including HCC (11-14). However, it is not understood whether serum level of PLGF is associated with prognosis of patient with HCC.

PLGF binds to only VEGF receptor-1 (VEGFR1) although VEGF binds to both VEGFR1 and VEGFR2 (15). PLGF stimulates endothelial migration and proliferation to develop angiogenesis through VEGFR1 in endothelium whereas VEGF stimulates mainly through VEGFR2 (15). A soluble form of VEGFR1 (sVEGFR1), a secretary protein generated through alternative splicing, lacks transmembrane and intracellular domain of VEGFR1 (16). Since sVEGFR1 can bind to both PLGF and VEGF, but not mediate intracellular signaling by these ligands, sVEGFR1 seems to be an intrinsic negative regulator for PLGF and VEGF (16). Forced expression of sVEGFR1 significantly suppresses tumor growth in a mouse orthotopic hepatoma model (17). However, the relationship between serum sVEGFR1 level and clinical features of HCC remains to be determined. There is growing evidence that the imbalance between PLGF and sVEGFR1 is implicated in development of preeclampsia (18). The lower ratio of PLGF to 
sVEGFR1, not the level of PLGF, showed a significant relationship with risk of preeclampsia to probably inhibit physiological PLGF-induced angiogenesis in placenta (18). Therefore, it is of great importance to estimate the ratio of PLGF to sVEGFR1 for assessment of intrinsic PLGF effect.

In this study, we measured the level of PLGF, sVEGFR1 and ratio of PLGF to sVEGFR1 in serum of patients with HCC and evaluated the relationship between these factors and outcome of patients with HCC. The information of this study is useful not only for assessing the prognostic values of these factors but also for considering the clinical implication of new therapy targeting PLGF and sVEGFR1.

\section{Materials and methods}

Patients and diagnosis. Between January 1999 and December 2000, 196 patients with untreated HCC were admitted to the Division of Gastroenterology, Department of Medicine, at Kurume University School of Medicine for HCC therapy. This was a cohort study comprising a collection of serial serum samples. This diagnosis of HCC was confirmed by needle biopsy or based on the findings of hypervascular liver masses with increased serum $\alpha$-fetoprotein (AFP) levels exceeding $400 \mathrm{ng} / \mathrm{ml}$, ultrasonography (US), computed tomography (CT), magnetic resonance imaging (MRI) and digital subtraction angiography were used for imaging techniques. In most of the patients, a percutaneous fine-needle aspiration biopsy was also performed under US guidance to confirm the diagnosis. Clinical staging was determined based on the TNM Classification of Malignant Tumors/International Union Against Cancer (UICC) classification system (19). The degree of tumor differentiation was determined histologically according to a modified Edmondson and Steiner Classification (20). On the basis of nuclear overcrowding, increased cytoplasmic basophilia and microacinar formation, tumors were defined as well differentiated, moderately differentiated (grade III) or poorly differentiated (grade IV).

Treatment and follow-up of patients. The following treatment criteria were used in this study: i) Percutaneous ethanol injection therapy (PEIT) or Radiofrequency ablation (RFA) was performed to allow US detection of $<4$ HCC lesions measuring $<3 \mathrm{~cm}$ each in size; ii) operation was performed for solitary lesions measuring $>3 \mathrm{~cm}$ or solitary lesions measuring $<3 \mathrm{~cm}$ that were undetectable by US; iii) Transarterial chemoembolization (TACE) was performed for multiple nodular HCC lesions where they numbered $>4$ or were $>3 \mathrm{~cm}$ in size; iv) hepatic arterial infusion chemotherapy (HAIC) was performed for multiple nodular lesions with major portal tumor thrombus (21); v) Patients with Child-Pugh grade $\mathrm{C}$ or distant metastases were treated with best supportive care (BSC).

After the initial treatment, the condition of each patient was carefully followed. Serum AFP and des- $\gamma$-carboxy prothrombin (DCP) concentrations were measured once every month. US and CT were performed every 3 months until 6 months post-treatment and every 6 months thereafter until 30 months post-treatment. The recurrence of HCC was confirmed by tumor enlargement or the appearance of new lesions in the imaging studies. When recurrence was suspected, an angiography or a percutaneous fine-needle aspiration liver biopsy was performed under US guidance. Subsequent treatments for recurrent HCC were selected according to tumor number and liver function. Therapy for a recurrent tumor included the following: i) PEIT or RFA was usually selected for recurrent HCC where tumors were $<2 \mathrm{~cm}$ and the number of nodules was $<3$; ii) TACE was selected for single nodules $>3 \mathrm{~cm}$ or for multiple nodules with unequivocal tumor stains; iii) HAIC was selected for multiple nodules with major portal tumor thrombus; iv) Patients with Child-Pugh grade C or distant metastases were treated with BSC. This study was closed in December 2008, or at the time of a patient's death. The possible causes of death were defined as: i) liver-related diseases: patients who died of tumor progression, liver failure or bleeding from esophageal-gastric varices or ii) others: patients who died of other diseases. If a patient had not been monitored in our hospital or in a related private Hepatology Clinic for $>1$ year, the patient was considered to have been lost to the follow-up procedure.

Measurement of serum PLGF and soluble VEGF receptor-1 ( $s$ VEGFR1) protein. Serial serum samples have been collected over many years at our hospital and maintained at $-20^{\circ} \mathrm{C}$. Serum PLGF and soluble VEGF receptor-1 (sVEGFR1) levels were measured with Quantikine ELISA kits (R\&D Systems, Inc. Minneapolis, MN, USA), according to the manufacturer's protocol (ref). Serum PLGF and sVEGFR1 levels values in preserved serum and fresh serum were compared to determine the influence of preservation at $-20^{\circ} \mathrm{C}$ using 10 samples. Because the standard deviation was $<10 \%$ (data not shown), we concluded that serum preservation at $-20^{\circ} \mathrm{C}$ did not affect the measured values.

Statistical analysis. Survival rates were determined by the Kaplan-Meier method and differences in the survival rates between the two groups were compared using the log-rank test. Analysis of multiple covariates of prognostic factors for the patient's background was performed with the Cox proportional hazards model. The $\chi^{2}$-test and Kruskal-Wallis rank test were used for comparisons of discrete variables. Statistical significance was defined as a P-value of $<0.05$. All statistical analyses were performed using the SPSS and SAS systems. The 14 factors examined were age at the diagnosis of HCC, gender, hepatitis B surface antigen ( $\mathrm{HBsAg})$, total bilirubin (TB), albumin (Alb), serum aspartate aminotransferase, serum alanine aminotransferase, platelet count, prothrombin time, Child-Pugh grade, serum PLGF, sVEGFR1, AFP, DCP and tumor stage. The cut-off value for the AFP and DCP levels was set at $200 \mathrm{ng} / \mathrm{ml}$ and $40 \mathrm{mAU} / \mathrm{ml}$, respectively. The initial treatment was classified into two groups of hepatectomy or PEIT and TACE or HAIC or BSC.

\section{Results}

Patient characteristics. One hundred and forty-eight patients were enrolled in this study and prognostic factors for HCC were prospectively analyzed by follow-up for a mean period of 3.44 years (median follow-up period, 2.5 years; range, 0.02-8.7 years). The patient characteristics are listed in Table I. Patient age ranged from 28 to 81 years (median: 66 years) and there were 105 males and 40 females. Of these patients, 
Table I. Characteristics of patients $(n=145)$.

\begin{tabular}{lc}
\hline Variables & $(\%)$ \\
\hline Age (years, median) & 65 \\
Gender (M/F) & $105 / 40$ \\
Positive for HBsAg and & \\
negative for anti-HCV (\%) & $11(7.6)$ \\
Positive for anti-HCV and & \\
negative for HBsAg (\%) & $115(79.4)$ \\
Negative for both HBsAg & \\
and anti-HCV (\%) & $17(11.7)$ \\
Positive for both Anti-HCV & \\
and HBsAg (\%) & 2 \\
Serum albumin (mg/dl) & 3.5 \\
Total bilirubin (mg/dl) & 0.9 \\
Serum aspartate aminotransferase (U/l) & 67 \\
Serum alanine aminotransferase (U/l) & 59 \\
Prothrombin time (\%) & 72 \\
Platelet count (x104) & $105 / 40$ \\
$\alpha$-fetoprotein (ng/ml) & \\
des- $\gamma$-carboxy prothrombin (mAU/ml) & 123.1 \\
PLGF/soluble VEGF receptor 1 & 0.168 \\
PLGF/soluble VEGF receptor 1(Ratio) & \\
Child Pugh grade (A/B + C) & $91 / 54$ \\
Tumor stage (1 + 2/3 + 4 ) & $(1.7-347190)$ \\
Initial treatment (hepatectomy or PEI & \\
or RFA/TAE or HAI/BSC) & \\
Average interval period (years) & \\
\hline
\end{tabular}

Tumor stage, TNM classification of malignant tumor sixth edition; PEI, percutaneous ethanol injection; TAE, trans arterial embolization; $\mathrm{HAI}$, hepatic arterial injection; BSC, best supportive care.

$11(11.7 \%)$ were positive for $\mathrm{HBsAg}$, but negative for antibodies to hepatitis $\mathrm{C}$ virus (anti-HCV), while 115 (79.4\%) patients were positive for anti-HCV, but negative for $\mathrm{HbsAg}$; two $(1.3 \%)$ patients were negative for both HBsAg and anti$\mathrm{HCV}$. Of all patients, $71(48.9 \%)$ were DCP-positive at the time of diagnosis, and $40(27.5 \%)$ patients were AFP-positive. Percutaneous fine-needle aspiration liver biopsy performed under US guidance was used to confirm the diagnosis of HCC in 27 of 145 patients. In 27 (18.6\%) patients, HCC was diagnosed pathologically. The remaining 118 (81.4\%) patients showed clinical features of HCC in the imaging study. Fifty-five patients underwent hepatectomy or PEIT or RFA, 79 patients were treated with TACE or HAIC and 11 patients received BSC.

Long-term outcomes. Follow-up data were obtained on 145 $(97.9 \%)$ patients while three patients $(2.1 \%)$ were lost during the follow-up period. In total, $112(77.2 \%)$ patients died from

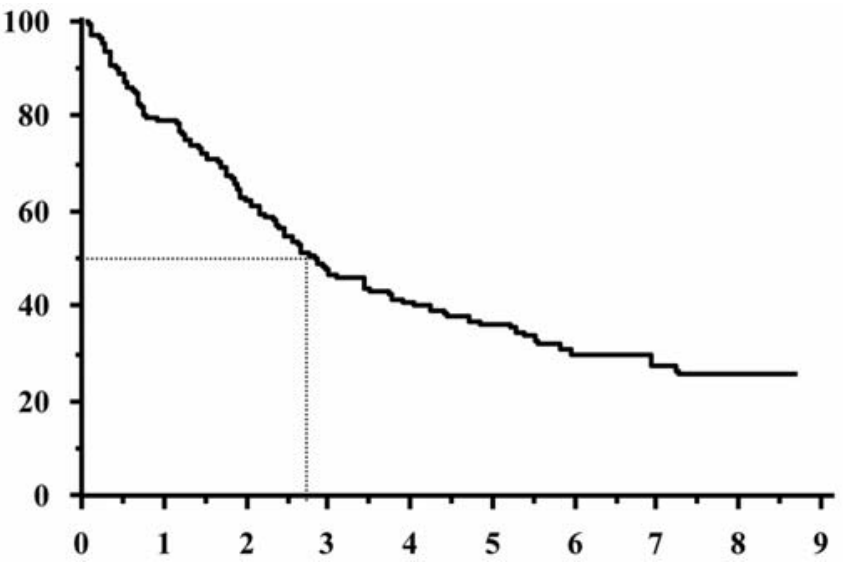

Figure 1. Overall survival rate of 145 patients who underwent initial treatment for hepatocellular carcinoma.

hepatic diseases which include tumor progression, hepatic failure and bleeding from esophageal-gastric varices.

Characteristics of the two groups classified according to $P L G F$, sVEGFRI and ratio of PLGF/sVEGFR1. The patients with $\mathrm{HCC}$ were divided into two groups at the time of diagnosis of HCC, respectively: i) 78 patients were high PLGF, 67 patients were low PLGF, ii) 73 patients were high sVEGFR1, 72 patients were low sVEGFR1, iii) 78 patients were high ratio of PLGF/sVEGFR1 (high PLGF/sVEGFR1 ratio), 67 patients were low ratio of PLGF/ sVEGFR1 (low PLGF/sVEGFR1 ratio). As shown in Table II, between high PLGF group and low PLGF group, there were no differences in regard to age, gender and etiology of cirrhosis. Tumor stage and Child-Pugh grade were significantly different between high sVEGFR1 group and low sVEGFR1 group. Child-Pugh grade and DCP levels were also correlated with serum sVEGFR1 level (Table III). High PLGF/sVEGFR1 ratio group was similar to low PLGF/sVEGFR1 ratio group with respect to all of the examined factors (Table IV).

Survival rates. Three-year survival rates for the 145 patients averaged $46.0 \%$ and the 5-year survival rate was $36.1 \%$ (Fig. 1). Fig. 2 shows that overall survival of patients in high PLGF group was lower than that of patients in low PLGF groups, which however failed to reveal statistical difference $(\mathrm{P}=0.122)$. The survival rate of high sVEGFR1 group was significantly lower than that of low sVEGFR1 group $(\mathrm{P}=0.0004)$ (Fig. 3). The overall survival of patients with low PLGF/sVEGFR1 ratio was significant poorer than that of those with high PLGF/sVEGFR1 ratio $(\mathrm{P}=0.03)$ (Fig. 4).

Univariate and multivariate analyses. The independent predictors of survival are summarized in Table V. According to univariate analysis, Age $>65$, Child-Pugh B or C grade, high sVEGFR1, low PLGF/sVEGFR1 ratio, AFP > $200 \mathrm{ng} / \mathrm{ml}$, DCP $>40 \mathrm{mAU} / \mathrm{ml}$, tumor stage 3 or 4 and initial treatment (TACE or HAIC or BSC) significantly correlated with survival. Multivariate Cox regression analyses were performed on these eight variables in the model. Five factors were found to be independently associated with survival: Age $>65$, Child-Pugh grade $\mathrm{B}$ or $\mathrm{C}$, low PLGF/sVEGFR1 ratio, AFP 
Table II. Comparison of characteristics of patients.

Variables

High PLGF

$(n=78)$

Low PLGF

Median age (years)

$(\mathrm{n}=67)$

Gender $(\mathrm{M} / \mathrm{F})$

66.9
$20 / 58$
6
61
10
1

66.8

Positive for HBsAg and negative for anti-HCV (\%)

$20 / 47$

Positive for anti-HCV and negative for HBsAg (\%)

5

Negative for both HBsAg and anti-HCV (\%)

Positive for both anti-HCV and HBsAg (\%)

Serum albumin $(\mathrm{mg} / \mathrm{dl})$

Total bilirubin (mg/dl)

Serum aspartate aminotransferase (U/l)

Serum alanine aminotransferase (U/l)

Prothrombin time (\%)

Platelet count $\left(\mathrm{x} 10^{4}\right)$

$\alpha$-fetoprotein (ng/ml)

Soluble VEGF receptor 1

PLGF

PLGF/soluble VEGF receptor 1 (Ratio)

Child Pugh grade $(\mathrm{A} / \mathrm{B}+\mathrm{C})$

Tumor stage $(1+2 / 3+4)$

Initial treatment

Tumor stage, TNM classification of malignant tumor sixth edition; PEI, percutaneous ethanol injection; TAE, trans arterial embolization; HAI, hepatic arterial injection; BSC, best supportive care.

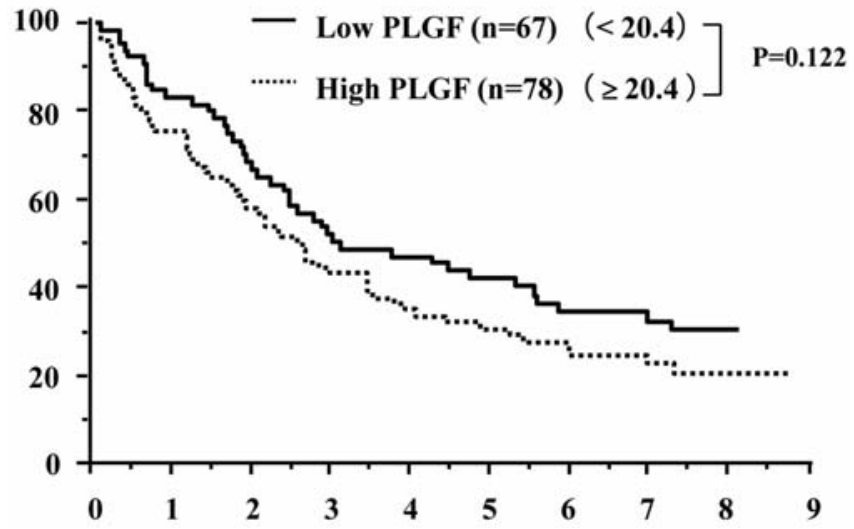

Figure 2. Survival rates according to the Kaplan-Meier method for the hepatocellular carcinoma patient groups defined by serum PLGF. The survival rate for the high PLGF group (dotted line; $n=78$ ) was lower than that for the low PLGF group (solid line; $\mathrm{n}=68$ ), but there was no significant differences.

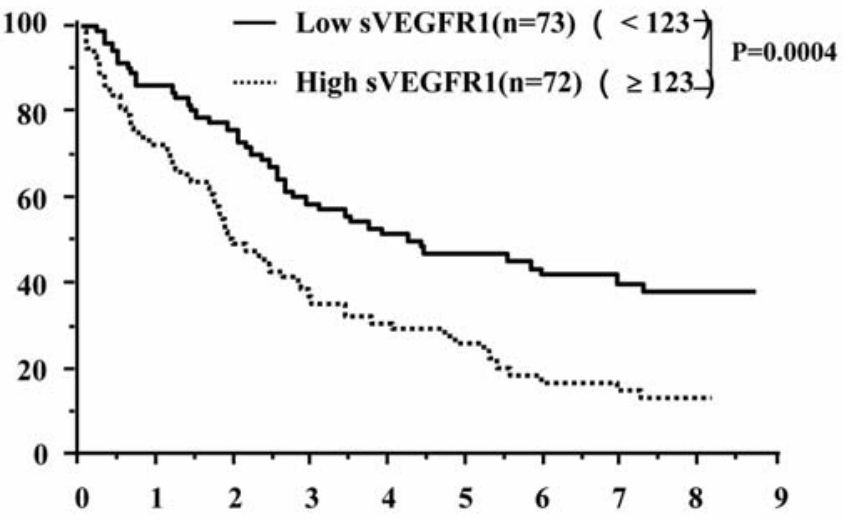

Figure 3. Survival rates according to the Kaplan-Meier method for the hepatocellular carcinoma patient groups defined by sVEGFR1. The survival rate for the high sVEGFR1 group (dotted line; $n=72$ ) was significantly lower than that for the low PLGF group (solid line; $\mathrm{n}=73)(\mathrm{P}=0.0004)$.
$>200 \mathrm{ng} / \mathrm{ml}$, tumor stages 3 or 4 and initial treatment (TACE or HAIC or BSC). The risk of death in patients in the low PLGF/sVEGFR1 ratio group was 1.87-fold higher than that in high PLGF/sVEGFR1 ratio group.

\section{Discussion}

The study reported herein is the first to estimate level of PLGF, sVEGFR1 and ratio of PLGF to sVEGFR1 in serum of 
Table III. Comparison of characteristics of patients.

\begin{tabular}{|c|c|c|}
\hline Variables & $\begin{array}{l}\text { High sVEGFR1 } \\
\qquad(\mathrm{n}=73)\end{array}$ & $\begin{array}{l}\text { Low sVEGFR1 } \\
\qquad(\mathrm{n}=72)\end{array}$ \\
\hline Median age (years) & 66.5 & 66.9 \\
\hline Gender $(\mathrm{M} / \mathrm{F})$ & $48 / 25$ & $57 / 15$ \\
\hline Positive for HBsAg and negative for Anti-HCV (\%) & 5 & 6 \\
\hline Positive for Anti-HCV and negative for HBsAg (\%) & 57 & 58 \\
\hline Negative for both HBsAg and anti-HCV (\%) & 11 & 6 \\
\hline Serum albumin $(\mathrm{mg} / \mathrm{dl})$ & 3.4 & 3.5 \\
\hline Total bilirubin (mg/dl) & 0.95 & 0.92 \\
\hline Serum aspartate aminotransferase (U/l) & 75 & 67 \\
\hline Serum alanine aminotransferase (U/l) & 59 & 60 \\
\hline Prothrombin time $(\%)$ & 71 & 75 \\
\hline Platelet count $\left(\mathrm{x} 10^{4}\right)$ & 9.7 & 10.5 \\
\hline$\alpha$-fetoprotein (ng/ml) & 37 & 46 \\
\hline des- $\gamma$-carboxy prothrombin $(\mathrm{mAU} / \mathrm{ml})$ & 59 & 111 \\
\hline Soluble VEGF receptor 1 & 131 & 111 \\
\hline Child Pugh grade $(\mathrm{A} / \mathrm{B}+\mathrm{C})$ & $32 / 41$ & $59 / 13$ \\
\hline Tumor stage $(1+2 / 3+4)$ & $48 / 25$ & $57 / 15$ \\
\hline Maximum tumor size $(\mathrm{cm})$ & 2.7 & 2.4 \\
\hline Vascular invasions (yes/no) & $15 / 58$ & $6 / 66$ \\
\hline \multicolumn{3}{|l|}{ Initial treatment } \\
\hline (Hepatectomy or PEI or RFA/TAE or HAI/BSC) & $24 / 39 / 10$ & $31 / 40 / 1$ \\
\hline
\end{tabular}

Tumor stage, TNM classification of malignant tumor sixth edition; PEI, percutaneous ethanol injection; TAE, trans arterial embolization; HAI, hepatic arterial injection; BSC, best supportive care.

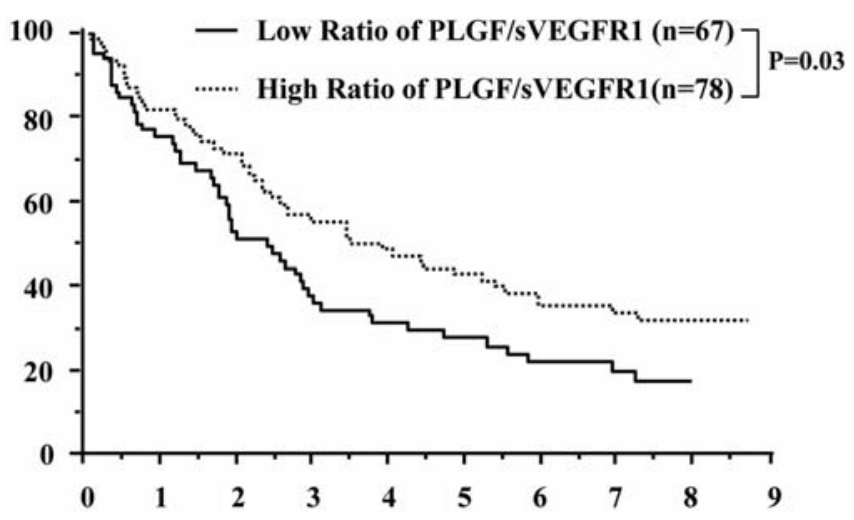

Figure 4. Survival rates according to the Kaplan-Meier method for the hepatocellular carcinoma patient groups defined by the ratio of PLGF to sVEGFR1. The survival rate for the high the ratio of PLGF to sVEGFR1 group (dotted line; $n=78$ ) was significantly better than that for the low the ratio of PLGF to sVEGFR1 group (solid line; $\mathrm{n}=67)(\mathrm{P}=0.03)$.

patient with $\mathrm{HCC}$ in order to evaluate the association between these factors and prognosis of HCC.

The expression of PLGF gene is highly regulated and PLGF protein is not detected in normal adult except for period of pregnancy. It is poorly understood in which type of cells PLGF protein is mainly produced. The level of PLGF expression is elevated when pathological neovascularization occurs in development of cancer or ischemic condition such as myocardial infarction. Recent study reports that expression of PLGF protein is elevated in several tumor tissues including HCC, and that intratumoral PLGF level is correlated with microvessel vascular density and poor outcome of cancer (11-14). PLGF-deficient mouse displays apparently normal growth process. Nevertheless, administration of neutralizing antibody for PLGF intensively inhibits tumor formation through decreasing tumor blood vessels without affecting healthy vessels in mouse model (10). PLGF has been thought to be a new indicator of pathological angiogenesis as well as a novel therapeutic target for cancer. In this study, we investigated whether serum PLGF level is associated with prognosis of patient with HCC. Serum PLGF level of patients with HCC is higher than those of chronic hepatitis and liver cirrhosis without HCC (data not shown). Overall survival rate of high PLGF group was poorer than that of low PLGF group, however there was no statistically significant difference. Serum level of PLGF was not an independent factor for prognosis of HCC. Recent studies showed that VEGF expression in HCC tumor tissue is decreased with increasing tumor size and that PLGF not VEGF expression levels correlate with early recurrence of HCC in patient with advanced disease $(5,11,22)$. In this study, high PLGF group was more progressed stage and elevated tumor marker than low PLGF group. Moreover, blockade of PLGF has been reported to markedly inhibit tumor formation which is resistant to VEGF inhibitor (10). These 
Table IV. Comparison of characteristics of patients.

\begin{tabular}{|c|c|c|}
\hline Variables & $\begin{array}{l}\text { Low ratio } \\
\qquad(n=67)\end{array}$ & $\begin{array}{l}\text { High ratio } \\
\quad(\mathrm{n}=78)\end{array}$ \\
\hline Median age (years) & 66.5 & 66.9 \\
\hline Gender $(\mathrm{M} / \mathrm{F})$ & $45 / 22$ & $60 / 18$ \\
\hline Positive for HBsAg and negative for anti-HCV (\%) & 8 & 3 \\
\hline Positive for anti-HCV and negative for $\mathrm{HBsAg}(\%)$ & 50 & 65 \\
\hline Negative for both HBsAg and anti-HCV (\%) & 9 & 8 \\
\hline Serum albumin $(\mathrm{mg} / \mathrm{dl})$ & 3.4 & 3.5 \\
\hline Total bilirubin (mg/dl) & 0.95 & 0.92 \\
\hline Serum aspartate aminotransferase (U/l) & 71 & 64 \\
\hline Serum alanine aminotransferase (U/l) & 59 & 60 \\
\hline Prothrombin time $(\%)$ & 72 & 74 \\
\hline Platelet count $\left(\mathrm{x} 10^{4}\right)$ & 9.7 & 10.5 \\
\hline$\alpha$-fetoprotein (ng/ml) & 37 & 46 \\
\hline des- $\gamma$-carboxy prothrombin (mAU/ml) & 59 & 111 \\
\hline Soluble VEGF receptor 1 & 131 & 111 \\
\hline PLGF & 17 & 25.5 \\
\hline PLGF/soluble VEGF receptor 1 (Ratio) & 0.126 & 0.217 \\
\hline Child Pugh grade $(\mathrm{A} / \mathrm{B}+\mathrm{C})$ & $39 / 28$ & $52 / 26$ \\
\hline Tumor stage $(1+2 / 3+4)$ & $49 / 18$ & $56 / 22$ \\
\hline Maximum tumor size (cm) & 2.5 & 2.6 \\
\hline Vascular invasions (yes/no) & $11 / 56$ & $10 / 68$ \\
\hline \multicolumn{3}{|l|}{ Initial treatment } \\
\hline (Hepatectomy or PEI or RFA/TAE or HAI/BSC) & $27 / 35 / 5$ & $28 / 44 / 6$ \\
\hline
\end{tabular}

Low ratio, low ratio of PLGF/sVEGFR1; high ratio, high ratio of PLGF/sVEGFR1; tumor stage, TNM classification of malignant tumor sixth edition; PEI, percutaneous ethanol injection; TAE, trans arterial embolization; HAI, hepatic arterial injection; BSC, best supportive care.

findings suggest that PLGF rather than VEGF might stimulate tumor blood vessels and be favorable therapeutic target in more advanced $\mathrm{HCC}$.

It has been also documented that PLGF is upregulated in inflammatory conditions. PLGF expression is higher in chronic active hepatitis patients without HCC than normal adults (23). Previous study showed that the pair of PLGF and its receptor VEGFR1 is expressed in sinusoidal endothelial cell of chronic hepatitis tissue (23). Moreover, PLGF has been shown to promote recruitment of CD34-positive cells, VEGFR1-positive endothelial progenitor cells, which are recruited in damaged liver tissue and plays an essential role for regeneration of liver (24). Besides, PLGF directly stimulates the production of proinflammatory cytokines interleukin-1ß, interleukin-8, monocyte chemoattractant protein-1 and VEGF in peripheral mononuclear cells (25). These findings indicate that serum PLGF level might reflect the degree of regeneration and inflammation in surrounding non-cancerous liver, in addition to development of pathological angiogenesis in HCC. Actually another group reported that serum PLGF level correlated with grade of fibrosis in chronic hepatitis and liver cirrhosis (26). In this study, high PLGF group had poorer liver function than low PLGF group in patients with HCC.

We next investigated the importance of serum sVEGFR1 level in prognosis of HCC. We found that higher level of
sVEGFR1 was associated with poor prognosis of patients with HCC. However, sVEGFR1 is not an independent prognostic factor for HCC. sVEGFR1 is generated through alternative splicing from VEGFR1 gene. sVEGFR1, lacking transmembrane domain and intracellular domain, can bind to PLGF but not mediate its intracellular signaling leading to angiogenesis (16). sVEGFR1 thus is regarded as an antiangiogenesis factor. It is little understood how expression of sVEGFR1 gene is regulated, although sVEGFR1 gene is abundant in all identified VEGFR 1 cDNAs from human endothelial cell (27). The expression of VEGFR1 has been shown to be up-regulated and correlated to microvascular density and histological differentiation of HCC (28). Since high sVEGFR1 group had a more advanced tumor stage than low sVEGFR1 group in this study, serum of sVEGFR1 at least partly might be derived from tumor tissues.

Expression of VEGFR1 is also observed in activated hepatic stellate cells and Kuppfer cells as well as sinusoidal endothelial cells in chronic hepatitis tissue (23). Accordingly, VEGFR1 signaling might partly drive activation of sinusoidal endothelial cells, stellate cells and kupffer cells leading to hepatic wound healing, fibrosis and inflammation as well as angiogenesis in HCC tissue. Serum level of sVEGFR1 was reported to be elevated in patient with chronic hepatitis although sVEGFR1 was not detected in normal adults (26). 
Table V. Univariate and multivariate analyses of survival in patients with hepatocellular carcinoma.

\begin{tabular}{|c|c|c|c|c|c|}
\hline \multirow[b]{2}{*}{ Variables } & \multirow[b]{2}{*}{ No. of patients } & \multicolumn{2}{|c|}{ Univariate analyses } & \multicolumn{2}{|c|}{ Multivariate analyses } \\
\hline & & $\begin{array}{l}\text { Hazard ratio } \\
\quad(95 \% \mathrm{CI})\end{array}$ & P-value & $\begin{array}{l}\text { Hazard ratio } \\
\quad(95 \% \mathrm{CI})\end{array}$ & P-value \\
\hline \multicolumn{6}{|l|}{ Age } \\
\hline$\geq 65$ & 92 & $1.81(1.17-2.81)$ & 0.007 & $2.11(1.28-3.48)$ & 0.003 \\
\hline \multicolumn{6}{|l|}{ Gender } \\
\hline Male & 105 & $0.85(0.55-1.33)$ & 0.48 & & \\
\hline \multicolumn{6}{|l|}{$\mathrm{HBV}$} \\
\hline HBsAg-positive & 11 & $1.44(0.24-1.42)$ & 0.59 & & \\
\hline \multicolumn{6}{|l|}{ Child } \\
\hline B or $\mathrm{C}$ & 45 & $3.70(2.45-5.60)$ & $<0.0001$ & $1.97(1.18-3.27)$ & 0.008 \\
\hline \multicolumn{6}{|l|}{ PLGF } \\
\hline$\geq 20.4$ & 78 & $1.36(0.91-2.03)$ & 0.12 & & \\
\hline \multicolumn{6}{|l|}{ Soluble VEGF receptor 1} \\
\hline$\geq 123$ & 72 & $1.53(1.03-2.27)$ & 0.032 & $0.99(0.60-1.64)$ & 0.99 \\
\hline \multicolumn{6}{|c|}{ PLGF/soluble VEGF receptor 1 (Ratio) } \\
\hline$<0.168$ & 67 & $1.53(1.03-2.27)$ & 0.032 & $1.87(1.18-2.95)$ & 0.007 \\
\hline \multicolumn{6}{|l|}{$\alpha$-fetoprotein } \\
\hline$\geq 200$ & 40 & $4.43(2.89-6.78)$ & $<0.0001$ & $3.04(1.68-5.49)$ & 0.0002 \\
\hline \multicolumn{4}{|l|}{ des- $\gamma$-carboxy prothrombin } & $1.26(0.69-2.28)$ & 0.44 \\
\hline \multicolumn{6}{|l|}{ Tumor stage } \\
\hline \multicolumn{6}{|l|}{ Initial treatment } \\
\hline TACE or HAI & 79 & $0.29(0.15-0.55)$ & 0.0002 & $0.16(0.07-0.34)$ & $<0.0001$ \\
\hline Hepatectomy or PEI or RFA & 55 & $0.065(0.03-0.14)$ & $<0.0001$ & $0.072(0.03-0.17)$ & $<0.0001$ \\
\hline
\end{tabular}

High sVEGFR1 group was poorer liver function than low sVEGFR1 group in this study. These findings suggest that the elevation of serum sVEGFR1 in patient with HCC might be attributed to both non-cancerous tissue and HCC tissue.

A previous study revealed that forced expression of sVEGFR1 inhibits tumor formation of HCC decreasing microvessel density in a mouse model (17). More recently, many studies revealed that the imbalance between sVEGFR1 and PLGF is implicated in preeclampsia (18). They suggest that over-abundance of sVEGFR 1 compared with PLGF inhibits angiogenesis in development of placenta. According to these findings, of interest is to evaluate whether the ratio of serum PLGF to sVEGFR1 correlates to outcome of HCC. Interestingly, lower ratio of PLGF to SVEGFR1 exhibited poor prognosis and independently predicted poor survival of HCC patients in this study. Previously, it has been shown that decreased expression of sVEGFR1 to VEGF in tumor and serum was involved in progression of tumors such as breast, pancreatic cancers (29-31). Higher ratio of VEGF to sVEGFR1 was correlated to poor survival and an independent prognostic indicator in those cancers. As sVEGFR 1 is regarded as inhibitor for both PLGF and VEGF, it is intriguing that the expression level of sVEGFR1 to PLGF or VEGF makes the difference between prognosis of HCC and those of breast and pancreas cancer. Prognosis of patients with HCC, in contrast to breast and pancreas cancer, is determined not only by progression of tumor but also liver function of surrounding hepatitis and cirrhosis. Thus, the difference is at least partly due to the abundance of sVEGFR1 for PLGF might affect restoration of hepatitis and cirrhosis tissue to inhibit liver regeneration, in addition to angiogenesis in HCC.

In conclusion, our present study revealed that the ratio of serum PLGF/serum sVEGFR1, not serum PLGF and serum sVEGFR1, is an independent factor for the prognosis of patients with HCC. The imbalance between PLGF and sVEGFR1 might be involved in outcome of patients with HCC affecting both progression of tumor and repair of surrounding hepatitis and cirrhotic liver. Thus, the balance of PLGF and sVEGFR1 should be addressed for considering an application of novel therapy targeting PLGF and sVEGFR1.

\section{Acknowledgements}

This study was supported in part by Health and Labour Sciences Research Grants for Research on Hepatitis from the Ministry of Health, Labour and Welfare of Japan (M.S.). We thank Ms. Masako Sinkawa for her excellent technical assistance, and Ms. Taeko Narisawa and Ms. Mieko Hatae for manuscript preparation. 


\section{References}

1. El-Serag $\mathrm{H}$ and Mason A: Rising incidence of hepatocellular carcinoma in the United States. N Engl J Med 340: 745-750, 1999.

2. Parkin D, Bray F, Ferlay J and Pisani P: Estimating the world cancer burden: Globocan 2000. Int J Cancer 94: 153-156, 2001.

3. Carr B: Hepatocellular carcinoma: current management and future trends. Gastroenterology 127: S218-S224, 2004.

4. Ng I, Poon R, Lee J, Fan S, Ng M and Tso W: Microvessel density, vascular endothelial growth factor and its receptors Flt-1 and Flk-1/KDR in hepatocellular carcinoma. Am J Clin Pathol 116: 838-845, 2001

5. Yamaguchi R, Yano H, Iemura A, Ogasawara S, Haramaki M and Kojiro M: Expression of vascular endothelial growth factor in human hepatocellular carcinoma. Hepatology 28: 68-77, 1998.

6. Li X, Tang Z, Zhou G, Lui Y and Ye S: Significance of vascular endothelial growth factor mRNA expression in invasion and metastasis of hepatocellular carcinoma. J Exp Clin Cancer Res 17: 13-17, 1998 .

7. Maglione D, Guerriero V, Viglietto G, Delli-Bovi P and Persico M: Isolation of a human placenta cDNA coding for a protein related to the vascular permeability factor. Proc Natl Acad Sci USA 88: 9267-9271, 1991.

8. Luttun A, Brusselmans K, Fukao H, et al: Loss of placental growth factor protects mice against vascular permeability in pathological conditions. Biochem Biophys Res Commun 295: 428-434, 2002

9. Luttun A, Tjwa M, Moons L, et al: Revascularization of ischemic tissues by PIGF treatment, and inhibition of tumor angiogenesis, arthritis and atherosclerosis by anti-Flt1. Nat Med 8: 831-840, 2002.

10. Fischer C, Jonckx B, Mazzone M, et al: Anti-PlGF inhibits growth of $\mathrm{VEGF}(\mathrm{R})$-inhibitor-resistant tumors without affecting healthy vessels. Cell 131: 463-475, 2007.

11. Ho M, Chen C, Lee $\mathrm{H}$, et al: Placenta growth factor not vascular endothelial growth factor $\mathrm{A}$ or $\mathrm{C}$ can predict the early recurrence after radical resection of hepatocellular carcinoma. Cancer Lett 250: 237-249, 2007.

12. Matsumoto K, Suzuki K, Koike H, et al: Placental growth factor gene expression in human prostate cancer and benign prostate hyperplasia. Anticancer Res 23: 3767-3773, 2003.

13. Parr C, Watkins G, Boulton M, Cai J and Jiang W: Placenta growth factor is over-expressed and has prognostic value in human breast cancer. Eur J Cancer 41: 2819-2827, 2005.

14. Chen C, Hsieh F, Cheng Y, et al: The significance of placenta growth factor in angiogenesis and clinical outcome of human gastric cancer. Cancer Lett 213: 73-82, 2004.

15. Landgren E, Schiller P, Cao Y and Claesson-Welsh L: Placenta growth factor stimulates MAP kinase and mitogenicity but not phospholipase C-gamma and migration of endothelial cells expressing Flt 1. Oncogene 16: 359-367, 1998.

16. Kendall $\mathrm{R}$ and Thomas $\mathrm{K}$ : Inhibition of vascular endothelial cell growth factor activity by an endogenously encoded soluble receptor. Proc Natl Acad Sci USA 90: 10705-10709, 1993.
17. Raskopf E, Dzienisowicz C, Hilbert T, et al: Effective angiostatic treatment in a murine metastatic and orthotopic hepatoma model. Hepatology 41: 1233-1240, 2005.

18. Levine R, Maynard S, Qian C, et al: Circulating angiogenic factors and the risk of preeclampsia. N Engl J Med 350: 672-683, 2004.

19. Wittekind C, Compton C, Greene F and Sobin L: TNM residual tumor classification revisited. Cancer 94: 2511-2516, 2002.

20. Edmondson $\mathrm{H}$ and Steiner P: Primary carcinoma of the liver: a study of 100 cases among 48,900 necropsies. Cancer 7: 462-503, 1954.

21. Nagaoka S, Yoshida T, Akiyoshi J, et al: Serum C-reactive protein levels predict survival in hepatocellular carcinoma. Liver Int 27: 1091-1097, 2007.

22. El-Assal O, Yamanoi A, Soda Y, et al: Clinical significance of microvessel density and vascular endothelial growth factor expression in hepatocellular carcinoma and surrounding liver: possible involvement of vascular endothelial growth factor in the angiogenesis of cirrhotic liver. Hepatology 27: 1554-1562, 1998.

23. Huang X, McCaughan G, Shackel N and Gorrell M: Up-regulation of proproliferative genes and the ligand/receptor pair placental growth factor and vascular endothelial growth factor receptor 1 in hepatitis C cirrhosis. Liver Int 27: 960-968, 2007.

24. Rafii S, Avecilla S, Shmelkov S, et al: Angiogenic factors reconstitute hematopoiesis by recruiting stem cells from bone marrow microenvironment. Ann NY Acad Sci 996: 49-60, 2003.

25. Selvaraj S, Giri R, Perelman N, Johnson C, Malik P and Kalra V: Mechanism of monocyte activation and expression of proinflammatory cytochemokines by placenta growth factor. Blood 102: 1515-1524, 2003.

26. Salcedo Mora X, Sanz-Cameno P, Medina J, et al: Association between angiogenesis soluble factors and disease progression markers in chronic hepatitis C patients. Rev Esp Enferm Dig 97: 699-706, 2005.

27. Kendall R, Wang $G$ and Thomas $\mathrm{K}$ : Identification of a natural soluble form of the vascular endothelial growth factor receptor, FLT-1, and its heterodimerization with KDR. Biochem Biophys Res Commun 226: 324-328, 1996.

28. Amaoka N, Saio M, Nonaka K, et al: Expression of vascular endothelial growth factor receptors is closely related to the histological grade of hepatocellular carcinoma. Oncol Rep 16: 3-10, 2006.

29. Bando H, Weich H, Brokelmann M, et al: Association between intratumoral free and total VEGF, soluble VEGFR-1, VEGFR-2 and prognosis in breast cancer. Br J Cancer 92: 553-561, 2005.

30. Banerjee S, Pancholi S, A'hern R, et al: The effects of neoadjuvant anastrozole and tamoxifen on circulating vascular endothelial growth factor and soluble vascular endothelial growth factor receptor 1 in breast cancer. Clin Cancer Res 14: 2656-2663, 2008.

31. Chang Y, Chang M, Wei S, et al: Serum vascular endothelial growth factor/soluble vascular endothelial growth factor receptor 1 ratio is an independent prognostic marker in pancreatic cancer. Pancreas 37: 145-150, 2008. 